\title{
Laryngeal histoplasmosis: an occupational hazard
}

\author{
Jian Woei $\underline{\text { Teoh }}^{1,2}$, MD, MRCS, Faridah $\underline{\text { Hassan }}^{1}$, MBBS, FRCS, Mohd Razif Mohamad Yunus ${ }^{2}$, MBBS, MS ORL-HNS
}

\begin{abstract}
Isolated laryngeal histoplasmosis is a very rare entity. It has variable clinical presentations that might mimic both benign and malignant lesions, and is usually associated with pulmonary and other disseminated forms of histoplasmosis. Herein, we report a case of primary laryngeal histoplasmosis without the involvement of other systems in a 70-year-old Chinese man, who previously worked as a miner. He presented with a history of hoarseness for two months, with no other associated symptoms. Direct laryngoscopy revealed irregularity of the posterior onethird of both vocal folds. Histopathological examination revealed the presence of Histoplasma capsulatum on periodic acid-Schiff and Grocott's methenamine silver staining. The lesion resolved after one month of oral itraconazole treatment. However, the patient had to complete six months of antifungal treatment to prevent recurrence.
\end{abstract}

Keywords: histoplasmosis, laryngeal infection, leucoplakia

\section{INTRODUCTION}

Histoplasmosis is an infection caused by the intracellular fungus, Histoplasma capsulatum (H. capsulatum), and usually involves the respiratory system, particularly the lungs. H. capsulatum thrives well in damp acidic soil and spreads through inhalation of fungal spores, which travel into the lungs before entering the reticuloendothelial system. ${ }^{(1)}$ Laryngeal histoplasmosis usually occurs in immunocompromised patients (e.g. human immunodeficiency virus [HIV]-infected individuals, patients on immunosuppressants and elderly persons) through dissemination of the fungus from the lungs to other physiological systems. Isolated laryngeal histoplasmosis is therefore rare. Clinical presentations of laryngeal histoplasmosis may range from hoarseness and dysphagia to dyspnoea, with weight loss and malaise. ${ }^{(2)}$ It may also present as leucoplakic lesions or exophytic masses. ${ }^{(2)}$

\section{CASE REPORT}

A 70-year-old Chinese man presented with a history of hoarseness for two months with no dyspnoea, dysphagia or odynophagia. He also had no constitutional symptoms or any history suggestive of tuberculosis infection.

Careful history taking revealed that the patient had been a heavy smoker for the past 50 years, and had been exposed to bird and bat droppings while working as a tin miner in the jungles for 30 years. Although he had retired ten years ago, he continued to go on regular hunting trips to the jungles after retirement. He had a history of diabetes mellitus (which was being treated with oral hypoglycaemic agents with good sugar control), rate-controlled atrial fibrillation and ischaemic heart disease.

Clinically, there was no respiratory distress, but the patient's voice was noted to be hoarse. There was no stridor, and neck and chest examinations were unremarkable. However, flexible nasopharyngolaryngoscopy showed that the mucosae at the posterior one-third of both vocal folds were irregular.

Screening for HIV was negative. While the patient's random blood glucose level was found to be mildly elevated, his renal profile was normal. As chest radiography showed a mild increase of left hilar opacity, computed tomography (CT) of the thorax was performed. However, CT of the thorax only showed minimal base pleural thickening, with clear lung fields bilaterally, and no evidence of consolidation, lung mass or nodule, bronchiectasis, or fibrosis changes.

Direct laryngoscopy under general anaesthesia revealed irregular bilateral vocal folds involving the whole length of both vocal cords, although there were no other abnormal findings. A biopsy sample was consequently taken. Histopathological examination showed small, fragmented and inflamed stratified squamous epithelial lining, with scanty underlying stroma. The stroma was heavily infiltrated by chronic inflammatory cells that were predominantly composed of macrophages, and contained multiple yeast forms of fungal bodies within the cytoplasm, which stained positive with Grocott's methenamine silver (Fig. 1) and periodic acid-Schiff (Fig. 2) stains. The squamous epithelial cells showed regenerative changes and no malignancy was seen. These features were consistent with histoplasmosis.

Intravenous (IV) amphotericin B was initiated at $0.5 \mathrm{mg} / \mathrm{kg} /$ day, and slowly tapered up. As the patient developed renal impairment after the second dose of IV amphotericin B, even though IV hydration was instituted prior to every dose of IV amphotericin B, a decision was made to withhold further treatment. Renal function improved after one week of hydration. However, serum creatinine remained mildly elevated. Therefore, the patient was given oral itraconazole $400 \mathrm{mg}$ once daily.

On follow-up one month after the initiation of oral itraconazole therapy, the patient's voice had improved and

${ }^{1}$ Otorhinolaryngology Department, Hospital Selayang, Selangor, ${ }^{2}$ Department of Otorhinolaryngology, Universiti Kebangsaan Malaysia, Kuala Lumpur, Malaysia Correspondence: Dr Teoh Jian Woei, Medical Officer, Department of Otolaryngology, Hospital Universiti Kebangsaan Malaysia, Jalan Yaakob Latiff, Bandar Tun Razak 56000, Kuala Lumpur, Malaysia. tjwoei@yahoo.com 


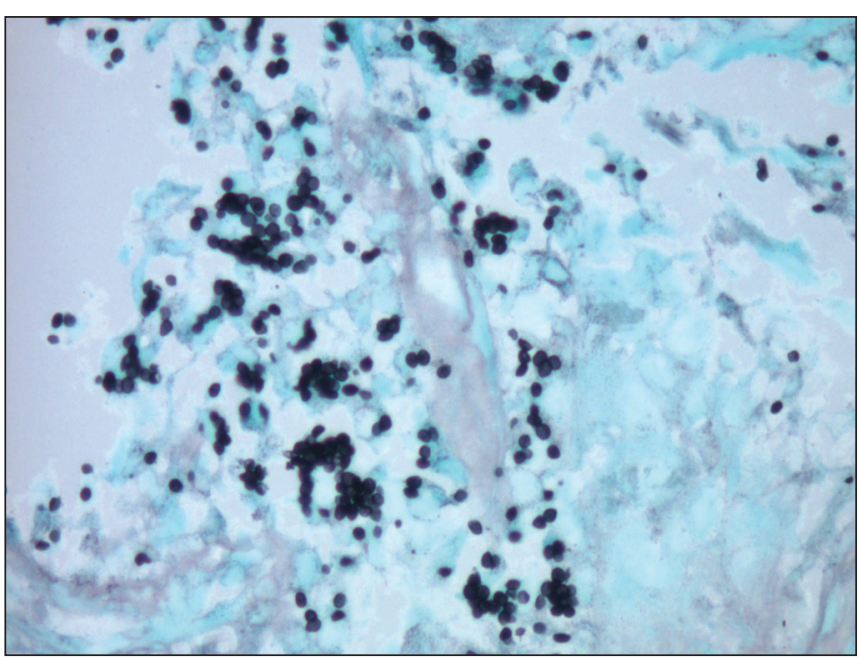

Fig. 1 Photomicrograph shows intracellular Histoplasma capsulatum (Grocott's methenamine silver, × 400).

the lesion over both of the vocal folds had disappeared. On the advice of an infectious disease specialist, oral antifungal treatment was continued for another five months.

\section{DISCUSSION}

Histoplasmosis is an infection caused by $H$. capsulatum, a dimorphic fungus that grows well in acidic soil with high nitrogen content. ${ }^{(1)}$ Bird and bat droppings enhance sporulation of $H$. capsulatum, thereby increasing its growth rate. However, fresh droppings on surfaces such as sidewalks have not been shown to contain H. capsulatum. ${ }^{(1)}$ Although bird droppings provide a nutrient source for the growth of the fungus, birds do not appear to be infected by it. ${ }^{(3)}$ Bats, however, can be infected by the fungus, and hence their droppings might contain the organism. ${ }^{(3)}$

Histoplasmosis is caused by the inhalation of $H$. capsulatum spores. As it cannot be transmitted from human to human, it is not contagious. ${ }^{(4)}$ Inhaled H. capsulatum spores, in the form of microconidia, is converted to the yeast form in lung parenchyma before being phagocytised by macrophages in the lungs at body temperature. ${ }^{(1)}$ Subsequently, in immunocompetent individuals, inflammation at the site of infection occurs, resulting in either caseating or noncaseating granuloma formation. However, in immunocompromised individuals or patients with weakened immune systems (e.g. patients with acquired immunodeficiency syndrome or malignancy, and patients on chemotherapy drugs, or high-dose or long-term steroid treatment), the infection will be disseminated to other systems. ${ }^{(5)}$ Construction workers, ${ }^{(2)}$ bridge inspectors or painters, ${ }^{(6)}$ farmers, ${ }^{(7)}$ as well as cave explorers ${ }^{(8)}$ are at risk of being infected by $H$. capsulatum.

Histoplasmosis primarily involves the lungs, and isolated histoplasmosis of the larynx is rare. To date, there are limited cases of primary laryngeal histoplasmosis reported in the literature compared to other laryngeal disorders. ${ }^{(9)}$ In most of the reported cases, laryngeal histoplasmosis presented with various clinical symptoms including hoarseness, dysphagia,

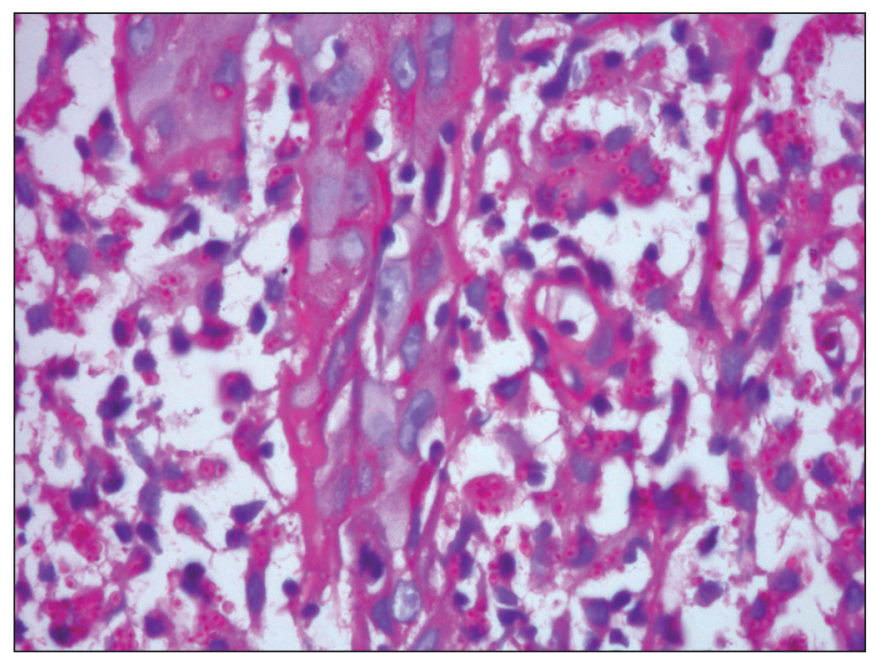

Fig. 2 Photomicrograph shows intracellular Histoplasma capsulatum (Periodic acid-Schiff, $\times 400$ ).

odynophagia, sore throat, dyspnoea, fatigue and weight loss. ${ }^{(9-12)}$ On direct laryngoscopy, features of the larynx range from irregular and thickened vocal folds, pearly white oedematous and necrotic vocal folds to ulcerated, solitary nodule and exophytic mass arising from the vocal folds. ${ }^{(9-12)}$

Swab and biopsy from the ulcer base can be sent for culture and histopathological examination. Special staining methods, such as those using Grocott's methenamine silver and periodic acid-Schiff stains, reveal small intracellular round-to-oval budding yeast at body temperature. Laryngeal histoplasmosis may also cause chronic inflammation with either caseating or noncaseating granuloma formation. Hence, tuberculosis (which also may present as caseating granuloma), paracoccidioidiomycosis (although this may present as granulomatous lesion with budding yeast - a 'mickey mouse' or 'steering wheel' appearance under the microscope is suggestive of Paracoccidioides spp.) and squamous cell carcinoma (chronic fungal infection might induce pseudoepitheliomatous hyperplasia that may mimic squamous cell carcinoma $^{(13)}$ may be the differential diagnoses of laryngeal histoplasmosis lesions.

History taking, clinical examination and investigations of patients with hoarseness must include a review of the various physiological systems, especially the respiratory system. Involvement of the mucosa of other parts of the aerodigestive tract $^{(13)}$ and lungs should be excluded. Sputum must be sent for investigation and chest radiography must be done. The patient should also be screened for immunocompromised conditions such as HIV infection. If the patient is immunocompromised, more extensive examinations need to be performed to establish or obviate disseminated histoplasmosis.

IV amphotericin B is the mainstay of treatment for histoplasmosis and is effective in $68 \%-92 \%$ of patients. ${ }^{(14)}$ The recommended dosage of IV amphotericin B for patients who require hospitalisation is $0.7-1.0 \mathrm{mg} / \mathrm{kg} /$ day, with the total dose to be administered over $2-4$ months being $35 \mathrm{mg} / \mathrm{kg}$. However, studies have shown that other oral antifungals are 
as effective as IV amphotericin B in patients with mild to moderate histoplasmosis - oral itraconazole (200-400 mg once daily) was effective in treating $85 \%-100 \%$ of patients with disseminated histoplasmosis, ${ }^{(15)}$ oral fluconazole (200-400 mg once daily) was effective in $86 \%$ of all patients, ${ }^{(15)}$ while oral ketoconazole (200-400 mg once daily) was effective in $56 \%-70 \%$ of patients. ${ }^{(15)}$

In summary, isolated laryngeal histoplasmosis is rare and might present with varied clinical presentations. For this reason, Histoplasma infection should be included in the differential diagnosis of miners and hunters presenting with laryngeal pathology. Biopsy specimens obtained during direct laryngoscopy should be sent for special staining and fungal culture. Besides IV amphotericin B, it is possible to use oral antifungals to treat isolated laryngeal histoplasmosis.

\section{ACKNOWLEDGEMENT}

The authors are grateful to Elaine Liew Li Fong for her help with the proofreading of the manuscript and for providing language assistance.

\section{REFERENCES}

1. Rippon JW. Histoplasmosis (histoplasmosis capsulati and histoplasmosis farciminosum). In: Medical mycology: the pathogenic fungi and the pathogenic actinomycetes. 3rd ed. Philadelphia: WB Saunders, 1988: 381-423.

2. Wheat LJ, Connolly-Stringfield PA, Baker RL, et al. Disseminated histoplasmosis in the acquired immune deficiency syndrome: clinical findings, diagnosis and treatment, and review of the literature. Medicine (Baltimore) 1990; 69:361-74.

3. George RB, Penn RL. Histoplasmosis. In: Sarosi GA, Davies SF, eds. Fungal diseases of the lung. Orlando: Harcourt Brace Jovanovich, 1986: 69-85.

4. Benenson AS. Control of communicable diseases manual. 16th ed. Washington: American Public Health Association, 1995: 237-40.

5. Johnson PC, Sarosi GA. Histoplasmosis. Semin Respir Med 1987; 9:145-51.

6. Sorley DL, Levin ML, Warren JW, Flynn JP, Gerstenblith. Bat-associated histoplasmosis in Maryland bridge workers. Am J Med 1979; 67:623-6.

7. Larsh HW. Histoplasmosis. In: DiSalvo AF, ed. Occupational mycoses. Philadelphia: Lea and Febiger, 1983: 29-41.

8. Gordon SM, Reines SS, Alvarado CS, et al. Disseminated histoplasmosis caused by Histoplasma capsulatum in an immunocompromised adolescent after exploration of a bat cave. Pediatr Infect Dis J 1993; 12:102-4.

9. Pochini Sobrinho F, Della Negra M, Queiroz W, et al. Histoplasmosis of the Larynx. Braz J Otorhinolaringol 2007; 73:857-61.

10. Subramaniam S, Abdullah AH, Hairuzah I. Histoplasmosis of the Larynx. Med J Malaysia 2005; 60:386-8.

11. Katoch P, Bhardwaj S. Primary Laryngeal Histoplasmosis. JK science: Journal of Medical Education \& Research [online], 2009; 11:89-90. Available at: www.doaj.org. Accessed April 4, 2011.

12. Le K, Daroca PJ Jr, Palacios E. Laryngeal histoplasmosis as a mimicker of squamous cell carcinoma: imaging and histologic findings. Ear Nose Throat J 2007; 86:662, 664.

13. Mills SE, Gaffey MJ, Frierson HF Jr. Benign squamous proliferations. In: Rosai J, Sobin LW, eds. Tumors of the upper aerodigestive tract and ear. Atlas of tumor pathology, 3rd series, fascicle 26. Washington: Armed Forces Institute of Pathology, 2000: 21-44.

14. Furcolow ML. Comparison of treated and untreated severe histoplasmosis. JAMA 1963; 183:121-7.

15. Wheat J, Sarosi G, McKinsey D, et al. Practice Guidelines for the Management of patients with Histoplasmosis. Infectious Diseases Society of America. Clin Infect Dis 2000; 30:688-95. 